\title{
Evolución de correlaciones cuánticas de dos qubits en una cavidad con disipación bifotónica
}

\section{Evolution of quantum correlations of two qubits in a cavity with biphotonic dissipation}

\author{
H. Molinares $\mathrm{a}^{*}$ \\ P. Villamil b* \\ V. Eremeev ${ }^{\mathrm{c}^{*}}$
}

Fecha de Recepción: 13.09.2018

Fecha de aceptación: 14.12.2018

DOI: https://doi.org/10.19053/01217488.v10.n1.2019.8411

\begin{abstract}
Resumen
En este trabajo se estudia la evolución de correlaciones cuánticas en un modelo Tavis-Cummings que consta de dos qubits y un único modo cuantizado del campo electromagnético. Se consideran procesos de emisión/absorción de un sólo fotón y de dos fotones correlacionados, para cada uno de los qubits. El modelo se estudia teniendo en cuenta disipaciones unifotónicas y bifotónicas, a través de una cavidad que interactúa con un reservorio térmico. La solución numérica de una ecuación maestra Lindblad nos permite realizar una descripción de la evolución de las correlaciones cuánticas, entrelazamiento cuántico y discordia cuántica, en función del tiempo para un estado atómico tipo superposición. Se muestra que los diferentes resultados de las correlaciones cuánticas, presentan comportamientos cualitativamente diferentes en su evolución.
\end{abstract}

Palabras clave: Correlaciones cuánticas, modelo Tavis-Cummings, ecuación maestra Lindblad, disipación unifotónica, disipación bifotónica.

a Universidad de Sucre, A. A. 406, Sincelejo, Colombia.

* Correo electrónico: molinareshugo@gmail.com

b Universidad de Sucre, A. A. 406, Sincelejo, Colombia.

* Correo electrónico: pablo.villamil@unisucre.edu.co

c Universidad Diego Portales, Sur 415, Santiago, Chile.

* Correo electrónico: vitalie.eremeev@udp.cl 


\begin{abstract}
In this work we study the evolution of quantum correlations in a Tavis-Cummings model that consists of two qubits and a single quantized mode of the electromagnetic field. The processes of emission / absorption of a single photon and of two correlated photons are considered for each of the qubits. The model is studied taking into account uniphotonic and biphotonic dissipations, through a cavity that interacts with a thermal reservoir. The numerical solution of a Lindblad master equation allows us to make a description of the evolution of quantum correlations, quantum entanglement and quantum discord, as a function of time for an atomic state type superposition. It is shown that the different results of the quantum correlations present qualitatively different behaviors in their evolution.
\end{abstract}

Key words: Quantum correlations, Tavis-Cummings model, Lindblad master equation, uniphotonic dissipation, biphotonic dissipation.

\section{INTRODUCCIÓN}

La interacción de un átomo con algunos modos del campo electromagnético cuantizado dentro de una cavidad, ha sido estudiada bajo el formalismo de la electrodinámica cuántica de cavidades (QED). Esto ha permitido avances en el procesamiento de la información cuántica [1, 2, 3], la teleportación cuántica [4], criptografía cuántica y la computación cuántica $[5,6]$. Sin embargo, parece muy difícil realizar una gran cantidad de operaciones debido a la interacción del sistema con el entorno [7, 8]. Debe destacarse que los experimentos QED han sido particularmente exitosos en la demostración de las características básicas de la mecánica cuántica, tales como, oscilaciones de Rabí y correlaciones cuánticas.

Cuando interactúan dos o más partes de un sistema cuántico, surge un tipo de correlaciones que no pueden ser explicadas clásicamente. A este fenómeno se le denomina entrelazamiento cuántico(EC) y fue introducido por E. Schrödinger en su intento por explicar el "experimento mental" propuesto por A. Einstein en la controversial publicación EPR [9]. Dos sistemas cuánticos $A$ y $B$ se entrelazan cuando ciertas propiedades del sistema A se correlacionan con las mismas propiedades del sistema $\mathrm{B}$, incluso en el caso en que ambos objetos están espacialmente lejos. Éste concepto se consideró casual, pero sólo hasta cuando John S. Bell hace público su teorema, la comunidad internacional evidencia éste fenómeno [10].

El EC es hoy un recurso necesario para realizar algunos algoritmos de información cuántica y una gran cantidad de esfuerzo se ha dedicado para estudiarlos y caracterizarlos. Sin embargo, se ha observado que el EC no cuantifica todas las correlaciones cuánticas. Entonces se ha introducido una serie de medidas de correlaciones cuánticas, la más popular es la discordia cuántica (DC) [11], que se define como la diferencia entre la información mutua del sistema y la información obtenida después de medir sobre una de las partes de dicho sistema.

En la actualidad se han desarrollado numerosas investigaciones acerca de la evolución de las correlaciones cuánticas ya que se han convertido en una herramienta esencial para la ingeniería de las comunicaciones cuánticas [12]. Se ha demostrado que los estados separables proporcionan aumento de velocidad computacional en comparación con los estados clásicos en algunos modelos de computación cuántica [13]. Se han realizado estudios acerca de la dinámica del entrelazamiento átomo-campo para dos átomos de dos niveles con transiciones de dos fotones degenerados que interactúan con único modo del campo electromagnético, teniendo en cuenta el efecto Stark [14], dinámica de correlaciones cuánticas y coherencia para dos átomos junto con un baño de campo escalar sin masa fluctuante [15], entrelazamiento en sistemas de estados arbitrarios de dos qubits [16] y discordia cuántica de dos qubits en estados $\mathrm{X}$ [17].

En éste artículo se estudia la dinámica de la evolución de las correlaciones cuánticas, entrelazamiento y discordia cuántica en un modelo tipo Tavis-Cummings con procesos multifotónicos, usando el formalismo de la ecuación maestra Lindblad y considerando fugas 
multifotónicas a través de la cavidad. Se examina la influencia del tipo de procesos de emisión/ absorción de fotones para los dos qubits, en la evolución de las correlaciones cuánticas. El artículo se ha organizado de la siguiente manera: En la sección 2 se realiza una descripción del modelo. Se presenta la forma de los Hamiltonianos y la ecuación maestra Lindblad teniendo en cuenta procesos multi-fotónicos. En la sección 3 se presentan los resultados obtenidos sobre el comportamiento evolutivo del entrelazamiento y la discordia cuántica. Finalmente, en el capítulo 4 se presentan las conclusiones.

\section{MODELO}

Se considera un sistema acoplado de dos qubits-cavidad con un único modo cuantizado, de frecuencia $\omega$, con pérdidas $\gamma$ por fuga de fotones a través de la cavidad, debido a la reflectividad imperfecta de los espejos. La frecuencia de transición $\omega_{0}$ es igual para ambos qubits y la interacción directa entre qubits es despreciable (ver figura 1).

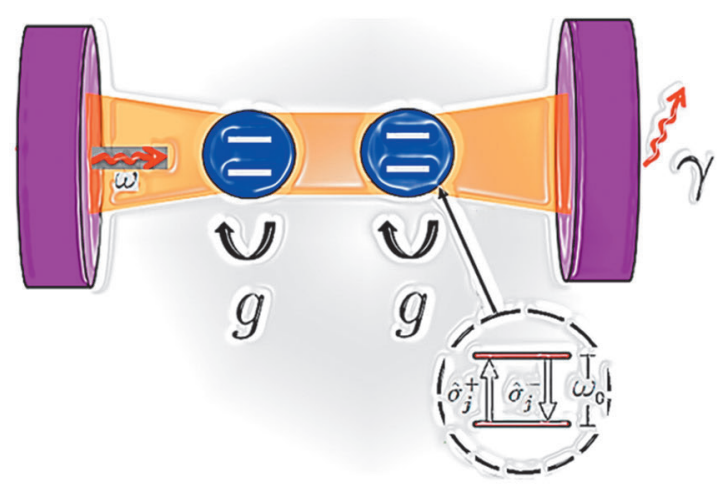

Figure 1. Representación del sistema acoplado qubitscavidad con un único modo cuantizado del campo electromagnético de frecuencia $\omega$, con pérdidas $\gamma$ por fuga de fotones a través de la cavidad hacia un baño térmico a temperatura $T$. Las flechas indican la transición entre los niveles del átomo $\mathrm{j}$ a través de los operadores $\hat{\sigma}_{j}^{+} \mathrm{y} . \omega_{0}$ es la frecuencia de transición de cada átomo

En este trabajo se consideran dos tipos de procesos de emisión/absorción de fotones para los qubits, procesos unifotónicos y procesos bifotónicos. En los procesos unifotónicos, el Hamiltoniano del sistema en la RWA, sin considerar pérdidas de fotones a través de la cavidad, viene dado por la siguiente expresión

$$
\begin{aligned}
\hat{\mathscr{H}}_{\mathrm{s}}^{(1 \mathrm{ph})}= & \hbar \omega \hat{a}^{\dagger} \hat{a}+\frac{\hbar \omega_{0}}{2} \sum_{j=1}^{2} \hat{\sigma}_{j}^{z} \\
& +\hbar g \sum_{j=1}^{2}\left(\hat{\sigma}_{j}^{+} \hat{a}+\hat{\sigma}_{j}^{-} \hat{a}^{\dagger}\right)
\end{aligned}
$$

Donde $\hat{\sigma}_{j}^{z}$ es el operador inversión de población del átomo $j, \hat{\sigma}_{j}^{ \pm}$es el operador de transición entre los niveles del átomo $j$, $\hat{a}\left(\hat{a}^{\dagger}\right)$ son los operadores de aniquilación (creación) del modo de la cavidad y $g$ es la constante de acoplamiento, asociada a la intensidad de la interacción coherente entre cada qubit y el modo cuantizado del campo electromagnético.

En el caso de procesos de emisión/absorción de bifotones correlacionados en los dos qubits, el Hamiltoniano del sistema en la RWA, sin considerar pérdidas de fotones es

$$
\begin{aligned}
\hat{\mathscr{H}}_{\mathrm{s}}^{(2 \mathrm{ph})} & =\hbar \omega \hat{a}^{\dagger} \hat{a}+\frac{\hbar \omega_{0}}{2} \sum_{j=1}^{2} \hat{\sigma}_{j}^{z} \\
& +\hbar g \sum_{j=1}^{2}\left(\hat{\sigma}_{j}^{+} \hat{a}^{2}+\hat{\sigma}_{j}^{-} \hat{a}^{\dagger 2}\right)
\end{aligned}
$$

Se define para los dos procesos un estado para el sistema usando la notación $|i\rangle=\left|A_{1}\right\rangle \otimes\left|A_{2}\right\rangle \otimes|C\rangle=\left|A_{1} A_{2} C\right\rangle, \quad$ donde $A_{j}=1,2$ corresponde a los estados atómicos, los cuales pueden ser $|e\rangle$ para el estado excitado y $|g\rangle$ para el estado base, $C$ representa el modo de la cavidad.

Las pérdidas mencionadas anteriormente se modelan usando la ecuación maestra en la forma de Lindblad, la cual describe las interacciones sistema-reservorio. Nuestro sistema de interés, qubits y cavidad, son parte de un sistema más grande, compuesto de una colección de osciladores armónicos cuánticos en equilibrio térmico a temperatura $T$. La evolución del sistema cerrado total se rige por el Hamiltoniano $\hat{\mathscr{H}}$ dado por

$$
\hat{\mathscr{H}}=\hat{\mathscr{H}}_{\mathrm{s}}+\hat{\mathscr{H}}_{\mathrm{r}}+\hat{\mathscr{H}}_{\text {int }}
$$

donde $\hat{\mathscr{H}}_{\mathrm{s}}$ es el Hamiltoniano del sistema sin pérdidas (ecuaciones 1 y 2 ), $\hat{\mathscr{H}}_{\mathrm{r}}$ es el Hamiltoniano del reservorio a temperatura finita y $\hat{\mathscr{H}}_{\text {int }}$ es el Hamiltoniano de interacción entre el sistema y el 
reservorio. Siguiendo el procedimiento estándar [18], se obtiene la ecuación maestra para el operador densidad reducido $\hat{\rho}_{\mathrm{s}}$

$$
\begin{aligned}
\dot{\hat{\rho}}_{\mathrm{s}}=-\frac{l}{\hbar} & {\left[\hat{H}_{\mathrm{s}}, \hat{\rho}_{\mathrm{s}}(t)\right] } \\
& +\sum_{k}\left(\hat{C}_{k} \hat{\rho}_{\mathrm{s}} \hat{C}_{k}^{\dagger}-\frac{1}{2}\left\{\hat{C}_{k}^{\dagger} \hat{C}_{k}, \hat{\rho}_{\mathrm{s}}\right\}\right)
\end{aligned}
$$

Donde $\hat{C}_{k}=\sqrt{\gamma_{k}} \hat{s}_{k}$ son los operadores colapso y $\hat{S}_{k}$ son operadores del sistema [19]. Para el modelo bajo estudio, la ecuación maestra tiene la forma

$$
\begin{aligned}
\dot{\hat{\rho}}_{\mathrm{s}}=- & \frac{l}{\hbar}\left[\hat{H}_{\mathrm{s}}, \hat{\rho}_{\mathrm{s}}\right] \\
& +\frac{1}{2}\left(2 \hat{C}_{1} \hat{\rho}_{\mathrm{s}} \hat{C}_{1}^{\dagger}-\hat{C}_{1}^{\dagger} \hat{C}_{1} \hat{\rho}_{\mathrm{s}}-\hat{\rho}_{\mathrm{s}} \hat{C}_{1}^{\dagger} \hat{C}_{1}\right) \\
& +\frac{1}{2}\left(2 \hat{C}_{2} \hat{\rho}_{\mathrm{s}} \hat{C}_{2}^{\dagger}-\hat{C}_{2}^{\dagger} \hat{C}_{2} \hat{\rho}_{\mathrm{s}}-\hat{\rho}_{\mathrm{s}} \hat{C}_{2}^{\dagger} \hat{C}_{2}\right)
\end{aligned}
$$

La forma de $\hat{C}_{1}$ y $\hat{C}_{2}$ para el caso de pérdidas unifotónicas, vienen dados por $\hat{C}_{1}=\sqrt{\gamma\left(1+\left\langle n_{t h}\right\rangle\right)} \hat{a} \quad$ y $\quad \hat{C}_{2}=\sqrt{\gamma\left\langle n_{t h}\right\rangle} \hat{a}^{\dagger}$ - Para pérdidas bifotónicas $\hat{C}_{2}=\sqrt{\gamma\left\langle n_{t h}\right\rangle} \hat{a}^{\dagger 2}$ y $\hat{C}_{2}=\sqrt{\gamma\left\langle n_{t h}\right\rangle} \hat{a}^{\dagger 2}$. $\gamma$ es la constante de acoplamiento entre el baño térmico y la cavidad, $\left\langle n_{t h}\right\rangle=1 /\left(e^{\hbar \omega / K_{b} T}-1\right)$ es el número promedio de bosones del reservorio a temperatura $T, \omega$ es la frecuencia del modo del campo electromagnético, h y $K_{b}$ son las constantes de Planck y Boltzmann, respectivamente.

\section{RESULTADOS}

En esta sección se presentan los resultados obtenidos de la evolución de las correlaciones cuánticas, concurrencia y discordia cuántica. Inicialmente se realiza una descripción del estado inicial del sistema global y se indican cada unos de los parámetros considerados.

Los átomos están en un estado inicial tipo superposición, el cual tiene la forma:

$$
\left|\psi_{\text {sup }}\right\rangle=\left|\psi_{\text {sup }}\right\rangle_{A_{1}} \otimes\left|\psi_{\text {sup }}\right\rangle_{A_{2}}
$$

donde $\left|\psi_{\text {sup }}\right\rangle_{A_{j}}=1 / \sqrt{2}\left(|e\rangle_{j}+|g\rangle_{j}\right) \operatorname{con}(j=1,2)$, es un estado tipo superposición asociado a cada uno de los átomos.
La expresión explícita para el estado coherente del campo electromagnético, viene dado en términos de los estados de Fock, así [20]:

$$
|\alpha\rangle=e^{-|\alpha|^{2} / 2} \sum_{n=0}^{\infty} \frac{|\alpha|^{n} e^{i n \phi}}{\sqrt{n !}}|n\rangle
$$

Aquí, se ha considerado el valor propio del estado coherente como $\alpha=\sqrt{2}$ y la dimensión para el espacio de Fock se ha acotado en $n=10$. El estado inicial para el operador densidad del sistema global tiene la forma:

$$
\hat{\rho}_{\mathrm{s}}=\left|\psi_{\text {sup }}\right\rangle\left\langle\psi_{\text {sup }}|\otimes| \alpha\right\rangle\langle\alpha|
$$

La expresión anterior indica que el estado inicial del sistema consta de dos átomos en un estado tipo superposición y el modo de la cavidad en un estado coherente. Se soluciona numéricamente la ecuación maestra y se obtiene el operador densidad atómico usando QuTiP [21]. A partir de allí, se determinan el entrelazamiento y la discordia cuántica.

La viabilidad experimental del sistema propuesto está relacionado con experimentos modernos en cavity-QED [22]. Los parámetros considerados en este estudio se normalizan a la frecuencia de la cavidad: $\omega=2 \pi \times 1 \mathrm{GHz}$. $\mathrm{La}$ desintonía entre la cavidad y los qubits $\Delta / \omega=0.1$, la constante de acoplamiento entre la cavidad y los qubits $g / \omega=0.3$, la constante de acoplamiento entre la cavidad y el baño térmico $\gamma / \omega=0.1$ y un número promedio de fotones térmicos $\left\langle n_{t h}\right\rangle=5$.

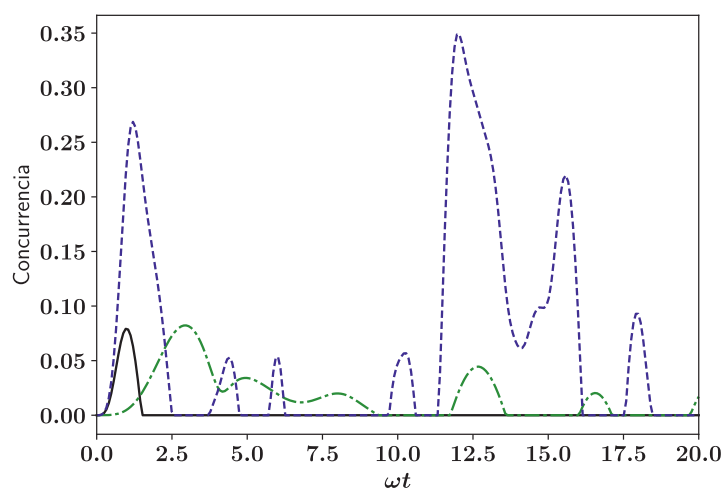

Figure 1. Evolución de la concurrencia para átomos en estado tipo superposición. Interacción unifotónica sin pérdidas (línea verde - línea - punto -), interacción bifotónica sin pérdidas (línea azúl - punteada -) e interacción bifotónica con pérdidas unifotónicas (línea negro - continua -). 


\subsection{Evolución de la concurrencia}

El grado de entrelazamiento entre los átomos puede ser determinado a través de la concurrencia $[16,23]$. En la figura 2 se muestra la evolución de la concurrencia de los dos qubits. La línea verde (línea- punto) y la línea azúl (punteada) corresponden a la evolución de la concurrencia sin pérdidas de fotones a través de la cavidad, mediante interacción unifotónica y bifotónica, respectivamente. La línea en color negro (continua) corresponde a la evolución de la concurrencia mediante interacción bifotónica con pérdidas unifotónicas, es decir, la pareja de fotones correlacionados pierde a uno de ellos que se escapa de la cavidad.

La evolución temporal de la concurrencia refleja la evolución temporal del entrelazamiento de los qubits. Para el caso sin pérdidas de fotones, las curvas corresponden al proceso de emisión de fotones por los átomos, las cuales se destruyen cuando los átomos absorben fotones. Como consecuencia de esto, hay entrelazamiento en algunos tiempos y de repente en algún momento finito comienza a acumularse un entrelazamiento.

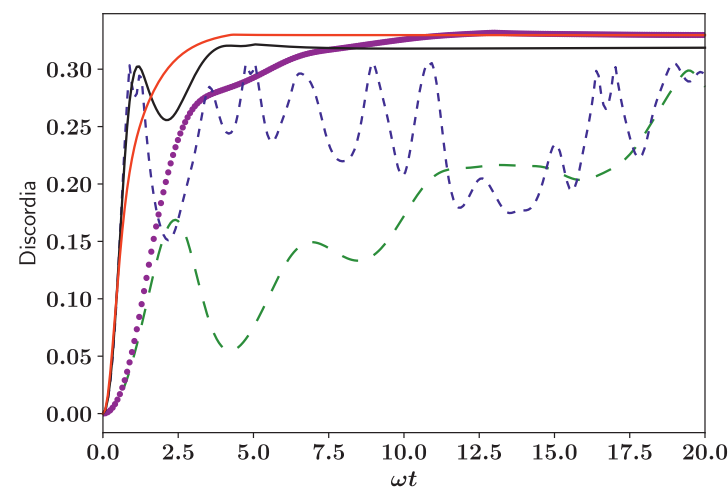

Figure 1. Evolución de la discordia cuántica para átomos en estado tipo superposición. Interacción unifotónica sin pérdidas (línea verde - línea - punto -), interacción unifotónica con pérdidas (línea morada - círculos -), interacción bifotónica sin pérdidas (línea azúl - punteada -), interacción bifotónica con pérdidas unifotónicas (línea negro - continua -) e interacción bifotónica con pérdidas bifotónicas (línea rojo - continua -).

Este efecto se conoce como "nacimiento repentino de entrelazamiento"("entanglement sudden birth") [23]. Para el caso de interacción bifotónica sin pérdidas, se puede observar que los átomos facilitan su entrelazamiento conforme evoluciona el tiempo hacia valores mayores con respecto a los procesos de un sólo fotón. En el caso de pérdidas unifotónicas, se logra el mayor grado de entrelazamiento de los dos qubits para un tiempo $\omega t \approx 1$ y posteriormente desaparece. Éste comportamiento se debe físicamente al hecho de que el entrelazamiento es una correlación muy sensible a la decoherencia, por lo tanto, el reservorio térmico la destruye rápidamente.

\subsection{Evolución de la discordia cuántica}

Otra forma de cuantificar las correlaciones cuánticas, es la discordia cuántica [11]. La figura 3 muestra la evolución de la discordia cuántica de los qubits. La línea verde (línea-punto) y la línea morada (círculos) corresponden a la evolución de la discordia cuántica del sistema, bajo interacción unifotónica sin pérdidas y con pérdidas unifotónicas, respectivamente. La línea azul (punteada) muestra el comportamiento de la discordia bajo interacción bifotónica sin pérdidas y las líneas negro (continua) y rojo (continua), corresponden a la evolución de la discordia cuántica del sistema bajo interacción bifotónica, con pérdidas unifotónicas y pérdidas bifotónicas, respectivamente.

Las curvas asociadas a la evolución de la discordia sin pérdidas, reflejan un comportamiento oscilatorio de mayor frecuencia en el caso de interacción bifotónica. Esta característica se debe a que los átomos y los bifotones correlacionados intercambian energía con una periódicidad mucho menor con respecto al caso de procesos unifotónicos. En la línea roja (continua), correspondiente a interacción bifotónica con pérdidas bifotónicas, se observa que para el modelo bajo estudio, la discordia cuántica alcanza su estabilidad de forma mucho más efectiva a diferencia de las disipaciones unifotónicas, tal como se puede ver en las curvas asociadas a las pérdidas (línea morada (círculos) y línea negro (continua)).

\section{CONCLUSIONES}

En éste trabajo hemos estudiado la evolución de correlaciones cuánticas en un modelo TavisCummings que interactúa con reservorio a temperatura finita. Examinamos la influencia 
del tipo de procesos de emisión/absorción de fotones para los dos qubits, en la evolución de las correlaciones cuánticas.

Los resultados indican que la discordia cuántica, a diferencia de la concurrencia, ha logrado la generación de un recurso de alta eficiencia a partir de este modelo, el cual se logra a través de la ecuación maestra Lindblad con interacción y pérdidas bifotónicas, tal como se puede apreciar en la curva roja (continua) de la figura 3, la facilidad que tienen los qubits de alcanzar una estabilidad mucho más rápida.

\section{AGRADECIMIENTOS}

H. Molinares, agradece a la Universidad de Sucre, Colombia, por el apoyo financiero durante la realización de éste estudio.

\section{REFERENCIAS}

[1] H. J. Kimble. The quantum internet. Nature, 2008.

[2] A. Romanelli, F. de Lima, R, Portugal y R, Donangelo. "The energy cost of quantum information losses", Nature, vol. 497, 2018.

[3] Z. Hu, X. Liang, J, Wang y Y. Zhang. "Quantum coherence and quantum correlation of two qubits mediated by a one-dimensional plasmonic waveguide". Opt. Express, vol. 24, no. 10, 2016.

[4] K.G. Paulson y S.V.M. Satyanarayana. "Bounds on mixedness and entanglement of quantum teleportation resources", Physics Letters A, vol. 381, 2017.

[5] M. Nielsen y I. Chuang. Quantum Computation and Quantum Information: 10th Anniversary Edition. Cambridge University Press, 2010.

[6] J. P. Aumasson. "The impact of quantum computing on cryptography". Computer Fraud and Security, vol. 2017, no. 6, 2017.
[7] I. de Vega y D. Alonso. "Dynamics of NonMarkovian open quantum systems". Rev. Mod. Phys., vol. 89, 2017.

[8] H. P. Breuer, E. Laine, J. Piilo, and B. Vacchini. "Colloquium: Non-Markovian dynamics in open quantum systems". Rev. Mod. Phys., vol. 88, 2016.

[9] A. Einstein, B. Podolosky y N. Rosen, "Can Quantum-Mechanical Description of Physical Reality Be Considered Complete?", Physical Review A, no. 47, 1935.

[10] J. S. Bell, "On the Einstein-Podolsky-Rosen paradox", Physics 1, 195-200, 1964.

[11] H. Ollivier and W. H. Zurek, "Quantum dis- cord: a measure of the quantumness of correlations", Physical Review Letters, vol. 88, no. 1, 2001.

[12] M. A. Nielsen y I. L. Chuang, Quantum Computation and Quantum Information, Cambridge University Press, Cambridge, 2000.

[13] Y.Zhang, X.Zou, Y.Xiay G. Guo, "Quantum discord dynamics in the presence of initial system cavity correlations", Journal of Physics B: Atomic, Molecular and Optical Physics, vol. 44, 2011.

[14] E. K. Bashkirov y M. S. Rusakovan, "Entanglement for two-atom TavisCummings model with degenerate twophoton transitions in the presence of the Stark shift", Optik - International Journal for Light and Electron Optics, vol. 123, no. 19, 1694 - 1699, 2012.

[15] Z. Huang y H. Situ. "Dynamics of quantum correlation and coherence for two atoms coupled with a bath of fluctuating massless scalar field", Annals of Physics, vol. 377, 2017.

[16] W. Wootters, "Entanglement of Formation of an Arbitrary State of Two Qubits", Physical Review Letters, vol. 80, no. 10, 1998. 
[17] S. Luo, "Quantum discord for two-qubit systems", Physical Review A, vol. 77, 2008.

[18] H. J. Carmichael, "Statistical Methods in Quantum Optics 1: Master Equation and Fokker-Planck Equations", Springer, New York, 1999.

[19] M. Weissbluth, Photon-Atom Interactions, Academic Press, INC, Stanford, California, 1989.

[20] M.O. Scully y M.S. Zubairy, "Quantum Optics”, Cambridge University Press, 2001.
[21] J. R. Johansson, P. D. Nation, F. Nori, “QuTiP 2: A Python framework for the dynamics of open quantum systems", Communication in Theorical Physics, vol. 184, 2012.

[22] S. Haroche y J. M. Raimond, "Exploring the Quantum: Atoms, Cavities and Photons", Oxford University, 2006.

[23] K. Zyczkowski, P. Horodecki, M. Horodecki y R. Horodecki, "Dynamics of quantum entanglement", Physical Review A, vol. 65, 2001. 